\title{
The involvement of J-protein AtDjC17 in root development in Arabidopsis
}

\section{Carloalberto Petti, Meera Nair and Seth DeBolt*}

Department of Horticulture, University of Kentucky, Lexington, KY, USA

\author{
Edited by: \\ Jan Traas, UMR \\ INRA-CNRS-ENSL-UCBL, France

\section{Reviewed by:} \\ Ján A. Miernyk, University of \\ Missouri, USA \\ Elena A. Vidal, Pontificia Universidad \\ Catolica de Chile, Chile

\section{*Correspondence:} \\ Seth DeBolt, Department of \\ Horticulture, University of Kentucky, \\ Plant Science Building, 1405 Veteran \\ Drive, N318 Agricultural Science \\ Center North, Lexington, \\ KY 40546-0312, USA \\ e-mail: sdebo2@email.uky.edu
}

In a screen for root hair morphogenesis mutants in Arabidopsis thaliana L. we identified a T-DNA insertion within a type III J-protein AtDjC17 caused altered root hair development and reduced hair length. Root hairs were observed to develop from trichoblast and atrichoblast cell files in both Atdjc17 and 35S::AtDJC17. Localization of gene expression in the root using transgenic plants expressing proAtDjC17::GUS revealed constitutive expression in stele cells. No AtDJC17 expression was observed in epidermal, endodermal, or cortical layers. To explore the contrast between gene expression in the stele and epidermal phenotype, hand cut transverse sections of Atdjc17 roots were examined showing that the endodermal and cortical cell layers displayed increased anticlinal cell divisions. Aberrant cortical cell division in Atdjc17 is proposed as causal in ectopic root hair formation via the positional cue requirement that exists between cortical and epidermal cell in hair cell fate determination. Results indicate a requirement for AtDJC17 in positiondependent cell fate determination and illustrate an intriguing requirement for molecular co-chaperone activity during root development.

Keywords: root hair, root patterning, development, heat shock protein, J-family, J-proteins

\section{INTRODUCTION}

During Arabidopsis thaliana L. Heyne root development, the distribution of hair cells has been extensively studied as a position-dependent developmental program. Here, the alternating emergence of trichoblast cells or root hair $(\mathrm{H})$ versus. atrichoblast cells or non-root hairs $(\mathrm{N})$ relies on positional information, whereby epidermal cells in direct contact with anticlinal cell walls of two underlying cortical cells acquire $\mathrm{H}$ cell fate and all others become N cells (Dolan et al., 1994; Dolan, 2006). This position-dependent cell fate determination at the root epidermis is reliant on a complex regulatory pathway involving genetic cell fate determinants and mobile transcriptional regulators (Kwak etal., 2005; Kwak and Schiefelbein, 2007). Here, SCRAMBLED (SCM), a leucine-rich receptor-like kinase (LRR-RLK) relays positional information underlying this pattern (Lee and Schiefelbein, 2002; Dolan, 2006; Kwak and Schiefelbein, 2008). Signaling through SCM establishes repression of the R2R3-MYB transcription factor WEREWOLF (WER) in $\mathrm{H}$ cells. As a result, the repression of WER in $\mathrm{H}$ cells causes increased levels of WER in $\mathrm{N}$ cells and in turn the levels of a regulatory complex consisting of transcriptional mediators WER-GLABRA3 (GL3)/ENHANCER OF GLABRA3 (EGL3)TRANSPARENT TESTA GLABRA1 (TTG1). The combined effect of these factors causes activation of direct targets including the $\mathrm{N}$-cell determinant and homeodomain-leucine-zipper transcription factor GLABRA2 (GL2; Kwak and Schiefelbein, 2008).

The complexity of cell fate determination in the epidermal layers is clearly evidenced by the large number of transcription regulators involved (Guimil and Dunand, 2006; Bruex et al., 2012), as well as by an increasingly large number of additional genes, e.g., auxin responsive, AXR2, AXR3 (Nagpal et al., 2000; Knox et al., 2003), KEU, which encodes the yeast Secl homolog, a key regulator of vesicle trafficking (Assaad et al., 2001), ROP2 a small Rho-plant GTPase implicated in cytoskeleton organization and cell polarity (Jones et al., 2002, 2006) and the ethylene oxide genes ETO1 and ETO2 involved in ethylene synthesis (Cao et al., 1999). Recently, a nuclear factor with homology to a heat shock factor (HSF; ten Hove et al., 2010), which encodes the SCHIZORIZA gene (Mylona et al., 2002), was also linked to radial patterning. Despite the potential for heat shock protein (HSP) or HSFs to play roles in root hair development and patterning, due to the obvious exposure to changing environmental conditions, genetic evidence for key genes remains poorly characterized.

A large number of genes encode the HSPs and HSFs. HSPs were named as heat response proteins (Ritossa, 1962; Tissiers et al., 1974), but since have been linked to a multitude of biological processes including microtubules stabilization, anti-apoptosis, refolding of a protein in non-native status, regulation of steroid hormone receptors (Kregel, 2002), protein translocation, protein folding reviewed by (Al-Whaibi, 2011), cell proliferation (Pechan, 1991) and at least with regard to fungi, HSPs are involved in signaling (Panaretou and Zhai, 2008). Among the HSPs the most studied are the HSP70 (Bakau and Horwich, 1998) and the DnaJ (Nakamoto and Vígh, 2007; Siddique etal., 2008). DnaJ is a member of the Hsp40 family of molecular chaperones, which is also known as J-protein family (Walsh et al., 2004). In Arabidopsis, the J-family encompass as many as 120 members classified in four sub-types (Rajan and D'Silva, 2009). J-protein type I, share all motifs found in a DnaJ a highly conserved N-terminal J-domain, followed by a glycine/phenylalanine rich region, a 
zinc-binding cysteine rich region and a variable C-terminal region. The remaining types display a simplified structure, with type II, missing the Zinc finger domain and type III displaying only the J-domain. Type IV, are J-like protein with a large similarity to the J-domain but missing the recognition motif HPD (Siddique et al., 2008; Rajan and D'Silva, 2009). DNAJ/ J-domain proteins are best know as co-chaperones working in client binary complex association with HSP70 (Minami et al., 1996; Miernyk, 2001; Qiu et al., 2006; Summers et al., 2009; Jelenska et al., 2010; Bekh-Ochir et al., 2013).

Herein, during a screen of T-DNA A. thaliana mutants for defective root hairs we identified that mutations in a type III Jprotein (AtDjC17) gene and we aimed to study the transcriptional and genetic features that contribute to root development.

\section{MATERIALS AND METHODS \\ PLANT MATERIAL AND GROWTH CONDITIONS}

Arabidopsis thaliana (L.) Heynh (Arabidopsis) ecotype Colombia0 was used in all experiments. The T-DNA insertional alleles [At5g23240, germplasm SALK_008678 (Atdjc17-1-1) and SALK_024726C (Atdjc17-1-2)] were obtained from the Arabidopsis Biological Resource Center (ABRC, Ohio State University). Seeds were surface sterilized and vernalized at $4^{\circ} \mathrm{C}$ for 2 days in the darkness prior to plating them on $1 / 2$ strength Murashige and Skoog (MS) basal salts medium (pH 5.7; Duchefa, Holland) solidified with $0.8 \%$ agar. Seeds were germinated under $16 \mathrm{~h}$ light; $8 \mathrm{~h}$ darkness conditions at a constant temperature of $22^{\circ} \mathrm{C}$. Seeds were plated as above described and plates were vertically positioned and incubated in dark grown $\left(22^{\circ} \mathrm{C}\right)$ condition. The phenotypes of Atdjc17-1-1 and Atdjc17-1-2 were compared to that of wild-type (WT) during plant growth and development. Plants were grown in MetroMix 360 (Sun Gro Horticulture) in a temperature controlled environmental chamber $\left(22^{\circ} \mathrm{C}\right.$; Adaptis, Conviron).

\section{GENOTYPING OF THE MUTANT LINES}

Homozygosity of the knockout lines Atdjc17-1-1/Atdjc17-1-2 was verified by polymerase chain reaction (PCR)-based genotyping, primers sequences are given in supporting information Table S1. Total plant DNA was extracted as previously described (Rogers and Bendich, 1985). For PCR purposes the DNA concentration was standardized to $100 \mathrm{ng} \mu \mathrm{l}^{-1}$ in Tris $\mathrm{pH} 8.0$ (10 mM).

\section{MICROSCOPY}

Imaging and quantitation of seedling phenotype employed fluorescence stereomicroscopy (Olympus MVX) and ImageJ (National Institutes of Health, Bethesda, MD, USA). Statistical analysis comparing Atdjc17 and WT plants used PRISM4 (Graphpad, La Jolla, CA, USA) and Minitab (Minitab Inc., USA). Seedling phenotypes, including root hair and epidermal patterning defects were examined consistently at 7-d post-germination. Seedlings were grown vertically in $1 / 2$ strength MS agar. Root hair length measurements were averaged across the entire root. To examine the pattern of epidermal development in a uniform spatial region of the root we documented cell area in the region covering $0.65 \mathrm{~mm}$ of root, initiating approximately
$2 \mathrm{~mm}$ above the root cap. Average cell length and area determinations for each trichoblast/atrichoblast cell used area measurement output after tracing the polygon via the freehand selection tool (ImageJ) and pixel-number ${ }^{2}$ converted to $\mu \mathrm{m}^{2}$. Due to the 3-dimensional nature of the root structure only those root hairs visible in the optical plane were counted. Transverse root sections were made as described (Hung et al., 1998) whereby roots were embedded in 3\% molten agarose and hand sectioned using double-edged razor blade. The sections were stained with calcofluor-white (Sigma, USA) stain and visualized under fluorescence stereomicroscope (Olympus MVX; DAPI filter). For $\beta$-glucuronidase (GUS) histochemical assay, staining solution was prepared according to (Guivarc'h et al., 1996). The seedlings were cleared, sectioned as above and counter stained with $0.05 \%$ Ruthenium red according to (Hassan et al., 2010) before visualization. Propidium iodide staining was performed as described in (Nawy et al., 2005). Accordingly 7-d post-germination seedlings were stained with $10 \mathrm{mgL}^{-1}$ propidium iodide for $30 \mathrm{~s}$ to $2 \mathrm{~min}$, rinsed and mounted on water. Microscopy was performed on an Olympus FV1000 laser scanning confocal microscope using a $63 \times$ N.A 1.4 water-immersion objective. The microscope is equipped with lasers for excitation wavelengths ranging from 405 to $633 \mathrm{~nm}$ and propidium iodide stain was excited using the DsRed setting in the Olympus Fluoview software (Olympus). All image processing was performed by using Olympus Fluoview software (Olympus) and ImageJ (W. Rasband, National Institutes of Health, Bethesda, MD, USA) software.

\section{CONSTRUCTION OF REPORTER AND OVEREXPRESSION LINES. SELECTION AND EXPRESSION ANALYSIS OF TRANSGENIC LINES}

The AtDjC17 transcript accumulation was assayed by fusing the AtDjC17 promoter to the GUS (Jefferson et al., 1987) reporter gene through a promoter::uidA fusion construct. A $1.5 \mathrm{~Kb}$ putative promoter region was PCR-amplified with the specific primers ATDJC17P-F/ATDJC17P-R (Supporting information Table S1) and the PCR amplified product was cloned into pCXPGUS ZeBaTA vectors (Chen etal., 2009). For overexpression studies, the open reading frame was PCR amplified from genomic DNA using primers ATDJC17G-F/ATDJC17G-R and the amplicon $(1.45 \mathrm{~Kb})$ was cloned into the pCXSN vector (Chen et al., 2009) under the constitutive expression of the Cauliflower mosaic virus (CaMV)-35S promoter (35S). Sequence verified clones were transformed by electroporation into Agrobacterium tumefaciens hypervirulent strain GVS3101. Arabidopsis plants were transformed (Clough and Bent, 1998) and homozygous alleles selected using the selectable marker hygromycin (25 $\mu \mathrm{g} / \mathrm{ml}$, Duchefa). Homozygous T3 plants from independent transformants were used in subsequent studies. T-DNA lines were complemented by restoring AtDjC17 under the control of the native promoter. The native promoter was PCR amplified and cloned within KpnI and HindIII sites of the pMDC32 vector replacing the $2 \mathrm{X} 35 \mathrm{~S}$ promoter. The full length AtDjC17 cDNA was cloned within the AscI and PacI sites completing the fusion cassette. For complementation, T-DNA lines were floral dipped and selected for hygromcyn resistance. T2/T3 generations were used for phenothypical characterization. 


\section{GENE EXPRESSION STUDIES}

Sterilized Atdjc17 and WT seed were germinated and grown vertically on $1 / 2$ strength MS agar plates in 16:8 light:dark conditions for 7-d. Root was rapidly excised from batches of approximately 200 seedlings using a surgical blade in aseptic conditions and snap frozen in liquid nitrogen. Total RNA was extracted using QIAGEN RNAeasy Plant mini kit and treated with DNAse I (Fermentas, LifeSciences) according to the manufacturer's instructions. Up to $2 \mu \mathrm{g}$ of the extracted total RNA was used for single stranded cDNA synthesis using High capacity cDNA reverse transcription kit (Applied Biosystems). The final volume was diluted fourfold and $2 \mu \mathrm{l}$ of the synthesized cDNA (100 ng) was used in the subsequent RT-PCR reactions. Quantitative real time PCR was conducted using Fast SYBR ${ }^{\circledR}$ Green Mastermix (Applied Biosystems) or HOTFIREPOL ${ }^{\circledR}$ EvAGreen ${ }^{\circledR}$ mastermix (OAK Biotechnologies LLC, USA) with StepOne ${ }^{\mathrm{TM}}$ Real-Time PCR system (Applied Biosystems). For the RT-PCR reaction the following conditions were used: 1 cycle of initial denaturation at $95^{\circ} \mathrm{C}$ for 10 or 15 min accordingly to the master mix employed, followed by 40 cycles of denaturation at $95^{\circ} \mathrm{C}$ for $15 \mathrm{~s}$ and annealing/extension at $60^{\circ} \mathrm{C}$ for $30 \mathrm{~s}$; followed by melting curve analysis. Actin 2 was used as internal control (Supporting information Table S1), with three-pooled biological replicates and three technical replicates. Primers for RT-PCR where possible were taken from referenced sources or designed using PRIMER3 (http://www.embnet.sk/ cgi-bin/primer3_www.cgi; Supporting information Table S1).

\section{RESULTS \\ MUTATIONS IN Atdjc17 CAUSED ALTERED ORGANIZATION OF ROOT HAIR POSITION IN ATRICHOBLAST VERSUS TRICHOBLAST CELL FILES}

In a screen for altered root hair (H-cell) occurrence, we identified a causal mutation in Atdjc17-1-1 (At5g23240). Motif analysis showed that AtDjC17 contained a J-domain motif and was broadly classified as a J-protein type III (Rajan and D'Silva, 2009). To further confirm the root hair phenotype, two alleles were isolated and correspond to Atdjc17-1-1 and Atdjc17-1-2. Homozygosity for the insertion of a T-DNA into the AtDjC17 exon was verified by PCR (see Supplementary material Figure S1 for insertion position). Further, AtDjC17 mRNA abundance was examined in WT as well as Atdjc17-1-1 and Atdjc17-1-2 plants by qRT-PCR and these data revealed no detectable AtDjC17 mRNA for either allele. Thus, we concluded that both Atdjc17-1-1 and Atdjc17-1-2 were null alleles.

$\mathrm{H}$-cells appeared in adjacent cell files rather than in alternating cell files in both mutant alleles (Atdjc17-1-1 and Atdjc17-1-2 respectively, Figure $\mathbf{1}$ and supplementary material Figure S2) described as irregular root $\mathrm{H}$ emergence. A $31 \%$ reduction in $\mathrm{H}$-cells was observed in a trichoblast cell file in Atdjc17-1-1 (Figures 1B-D). As expected, 100\% of H-cells were identified in the trichoblast cell file in WT roots $(n=10$ seedlings; Figures 1A-D). In Atdjc17-1-1 we observed H-cells in atrichoblast cell file (approximately 19.5\% H cells), whereas no $\mathrm{H}$ formation was observed in atrichoblast cell files in WT. Alongside these data we also observed a quantitative increase in the distance between adjacent H-cells in a cell file in Atdjc17 alleles compared with WT $(217.9 \pm 6.6 \mu \mathrm{m}$ for Atdjc17-1-1 and $173.4 \pm 5.8 \mu \mathrm{m}$ for WT, $P<0.05$; Figure $2 \mathrm{H}$ ). Accounting for this phenotype was an observed increase in trichoblast cell area for Atdjc17 mutants (Atdjc17-1-1: $2916 \pm 161.6 \mu \mathrm{m}^{2}$; WT: $\left.2384 \pm 104.2 \mu \mathrm{m}^{2}, P>0.05\right)$, indicative of an expansion defect.

To query whether increasing the transcript abundance for Atdjc17 would influence root epidermal patterning phenotype, we expressed a 35S::AtDjC17 in WT plants. This resulted in a transgenic plant with 3.5-fold increase in AtDjC17 transcript in the roots. Visual examination of the 35S::AtDjC17 roots also revealed irregular H-cell occurrence compared with WT. In an opposite fashion than was observed in Atdjc17 mutants, the 35S::AtDjC17 displayed a $12.5 \%$ reduction in $\mathrm{H}$-cells in a trichoblast cell file (Figures 1C-D) as compared to 31\% in Atdjc17-1. Average occurrence of $\mathrm{H}$-cells in the atrichoblast file was 34\% (Figure 1E) compared to the $19.5 \%$ in Atdjc17. Additionally, 35S::AtDjC17 displayed a significant increase in the number of $\mathrm{H}$-cells calculated per $0.65 \mathrm{~mm}$ of root length i.e., $19.4 \pm 0.7$ (Figure 2G, $P<0.05)$ when compared with the values determined for the WT (Figures 2A-G) and Atdjc17-1-1 (Figures 2B-G). This increase was also paralleled by a reduction of the distance between root H-cells $(135 \pm 2.9 \mu \mathrm{m}, P<0.05$; Figure $2 \mathbf{H})$ as compared to WT and to the Atdjc17 mutants. These data support the requirement for AtDjC17 in determining the correct positional distribution of H-cells among epidermis cells in the Arabidopsis root.

To further confirm that observed phenotypes in Atdjc17 were in response to dysfunction in $A t D j C 17$ we complemented the T-DNA mutant with the AtDjC17 driven by its native promoter (2000 bp upstream of initiation codon). The resulting complementation line was not discernible from the WT with respect to H-cell frequency and position (Supplementary Figure 3). These data were consistent with the observed phenotypes linked to AtDjC17.

\section{VISUAL EXAMINATION OF proAtDjC17::GUS TRANSCRIPT REVEALED LOCALIZATION TO THE STELE CELLS}

To visually determine where the AtDjC17 transcript was expressed during root development we generated a $\beta$-glucuronidase (GUS) reporter fused to the AtDjC17 promoter. Microscopic examination of 7-day old seedlings indicated a spatially discreet zone of transcript coincident with the stele and no visible abundance in cortical, endodermal or epidermal cell files (Figures 3A,B). Cross referencing of the stele expression for proAtDjC17::GUS with the cell sorting approach taken by Brady et al. (2007) was not conclusive as $A t D j C 17$ was expressed found to be expressed in low levels in the stele tissue. Transverse sections of stained and cleared seedlings confirmed that transcript was localized principally in the stele (Figure 3C). To also explore whether or not AtDjC17 transcript abundance was stress dependent or independent in a cell type specific manner, we imposed various stress regimes on the transgenic plants expressing proAtDjC17::GUS. These results revealed no shift in expression under stress.

\section{TRANSCRIPT ANALYSIS REVEALS DIFFERENTIAL EXPRESSION IN ROOT PATTERNING GENES IN Atdjc17-1 and 35S::AtDjC17 R00TS VERSUS WT}

The expression levels of known epidermal and radial patterning genes was explored. Here, we examined the LRR-RLK 


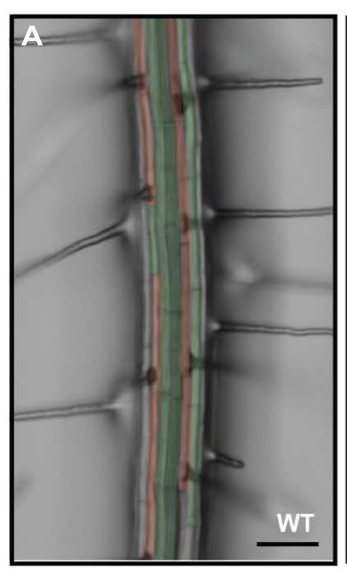

D

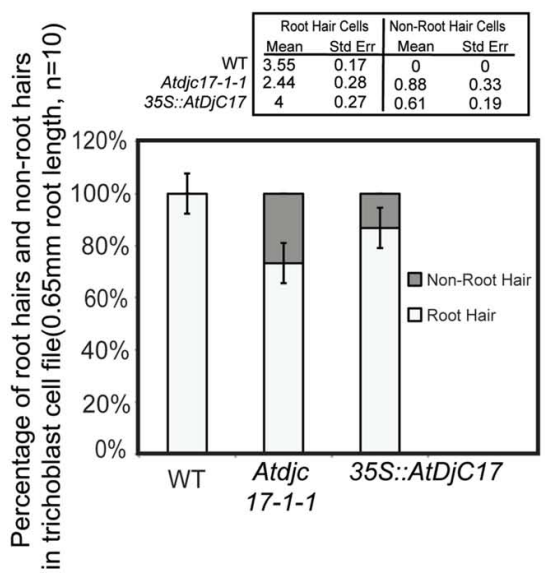

FIGURE 1 | Trichoblast and atrichoblast cells are arranged randomly in Atdjc17 and overexpressor mutants. (A-C) False colored stereomicroscope images of wild-type (WT; A), Atdjc17-1-1 (B) and 35S::AtDjC17 (C) illustrate the patterning of root hair cells (red) and non-root hair cells (green) in cell files. Stars represent ectopic root
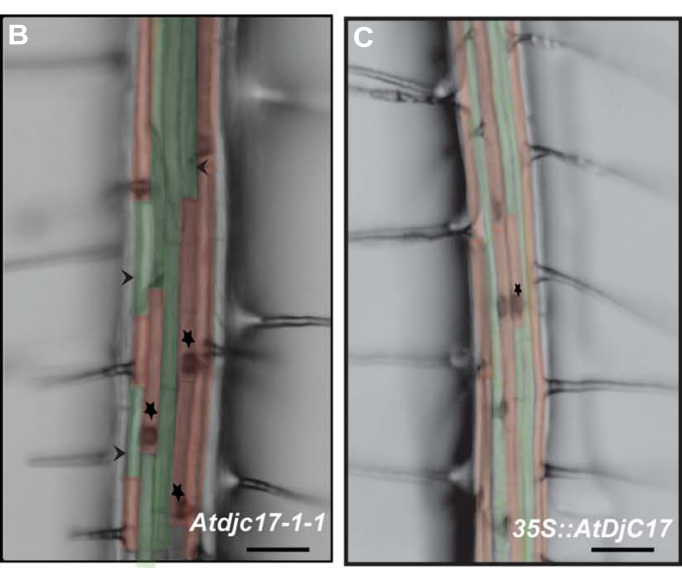

E

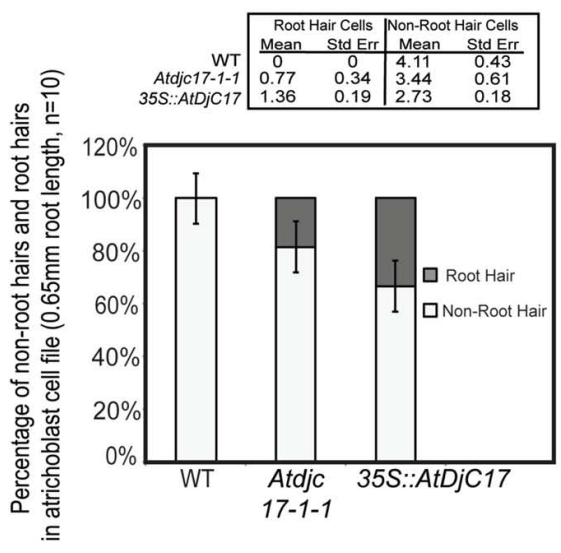

hairs in an otherwise atrichoblast cell file. Arrowheads represent the presence of non-root hair in an otherwise trichoblast cell file in Atdjc17-1-1. (D,E) Root hair and non-root hair cells in trichoblast and atrichoblast cell files were counted in WT, Atdjc17-1-1 and overexpressor 35S::AtDjC17.
(SCM), the bHLH transcription factors GLABRA3 (GL3) and EGL3, the R2R3-MYB transcription factor (WER), the small single-repeat R3-MYB transcription factor CAPRICE (CPC), the WD-repeat TTG1, the WRKY transcription factor TRANSPARENT TESTA GLABRA2 (TTG2), the homeodomain-leucinezipper transcription factor GLABRA 2 (GL2), the basic-leucine zipper transcription factor SCARECROW (SCR), the transcription factor $S H R$, and zinc finger proteins JACKDAW (JKD) and MAPGPIE (MGP; Galway et al., 1994; Di Laurenzio et al., 1996; Masucci etal., 1996; Wada etal., 1997, 2002; Lee and Schiefelbein, 1999; Helariutta et al., 2000; Schellmann et al., 2002; Sabatini et al., 2003; Zhang etal., 2003; Bernhardt et al., 2005; Koshino-Kimura etal., 2005; Ishida et al., 2007, 2008; Kwak and Schiefelbein, 2007; Welch et al., 2007; Hassan et al., 2010) in Atdjc17 alleles compared with WT. TTG2 amongst the epidermal patterning regulators and $J K D$ and $M G P$ involved in radial and epidermal patterning were not differentially expressed when compared to WT (Figure 4). Results showed a significant $(P \leq 0.05)$ up-regulation of GL3, SCM, EGL3, WER,
CPC, and TTG1 as compared to WT (Figure 4). The principle exceptions were $S C R$ and $S H R$, which were down-regulated $(P<0.05$, Figure 4). This trichoblast and atrichoblast specific transcripts increased abundance is consistent with both irregular H-cell development and reduced frequency of H-cells. $S C R$ transcript was down-regulated in the Atdjc17 mutant root (Figure 4) moreover SCR has been shown to cause a loss and coupling of endodermal and cortical cell layers (Di Laurenzio et al., 1996). However, mutants with loss in the AtDjC17 gene product did not exert as dramatic effects as other characterized mutants such as shr, as evidenced by agarose embedded hand-sections or propidium iodide staining (Figure 5). This down-regulation of SCR transcript likely resulted from an up or downstream regulation, such as $S H R$, which was also downregulated in Atdjc17. Nevertheless, a cell division increase was quantified in cortical (Increased cell division: Atdjc171-1/1-2: 9 cells/section \pm 1 vs. WT 8 cells/section $\pm 0, n=15)$ and endodermal layers (Increase in cell frequency in endodermal layer Atdjc171-1/1-2: 9 cells/section \pm 1 vs. WT 8 cells/section \pm 0 , 

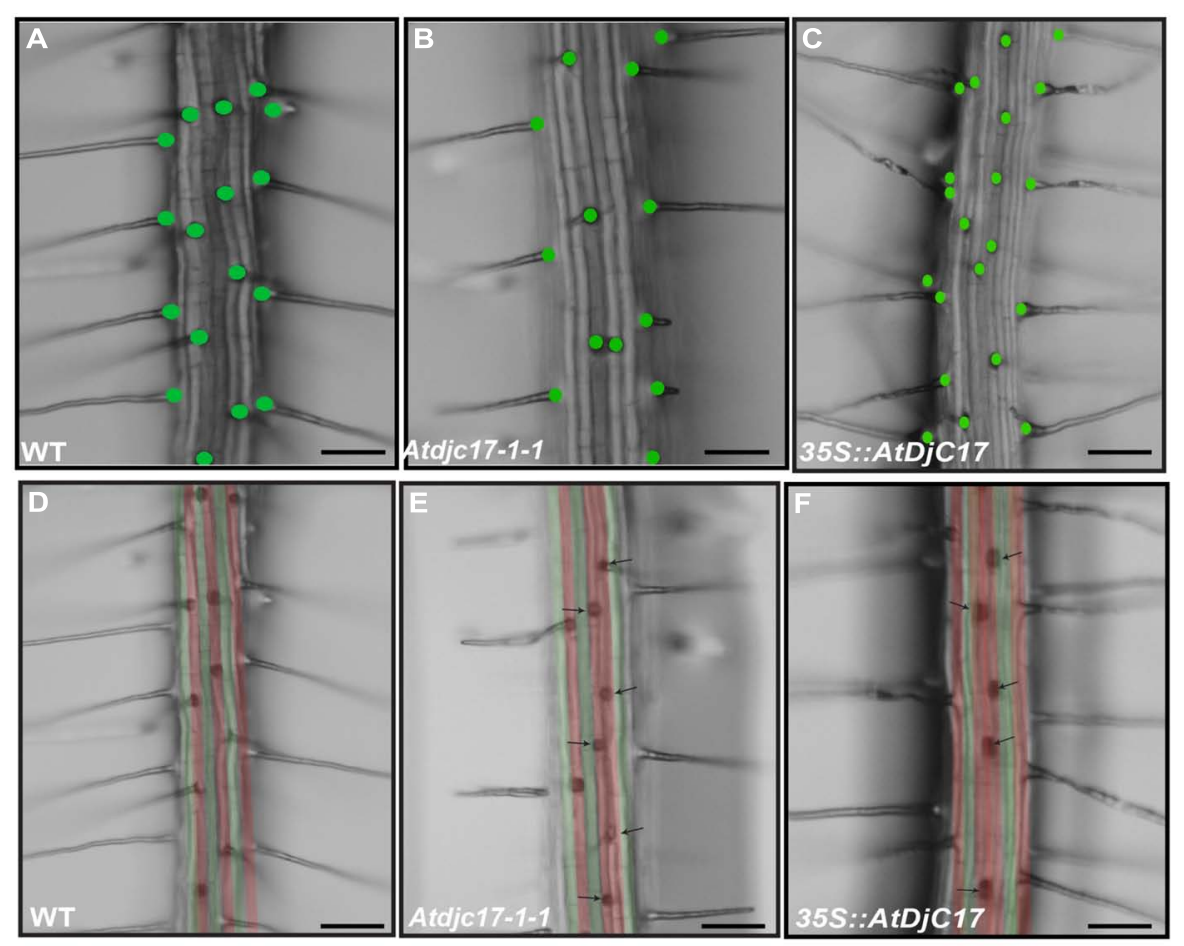

G
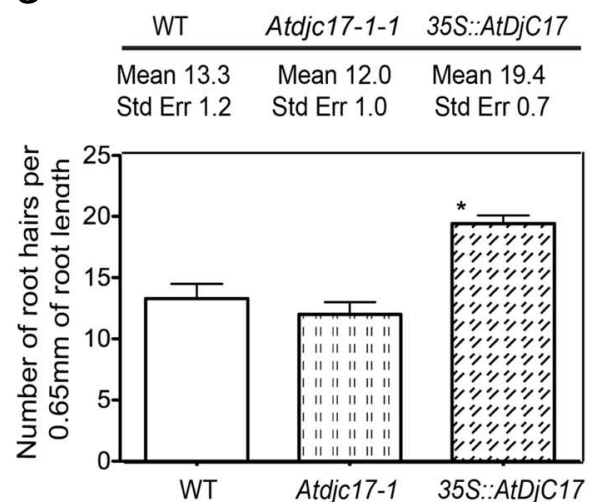

FIGURE 2 | Mutations and overexpression in ATDJC17 cause root hair alteration and ectopic root hair production. (A-C) Variation in number of root hairs is illustrated in Atdjc17-1-1 and 35S::AtDjC17 mutants as compared to WT plants. Green dots highlight the presence of root hair at the site. (G) Average number of root hairs determined from a total of 10 roots. An area approximately $2 \mathrm{~mm}$ from the root cap was chosen for the comparison covering $0.65 \mathrm{~mm}$ root length. (D-F) Stereomicroscope images of WT (D),
H

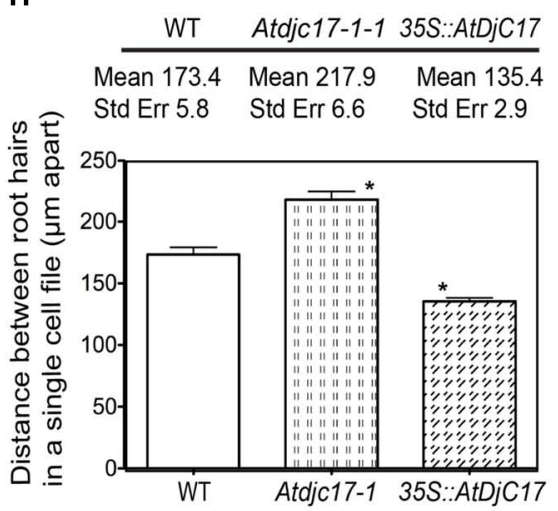

Atdjc17-1-1 (E) and 35S::AtDjC17 (F) roots false colored to show the trichoblast (red) and atrichoblast (green) cell files. Arrows in Atdjc17-1-1 and 35S::AtDjC17 mutant highlights the presence of two trichoblast cell files adjacent to each other illustrating the presence of ectopic root hair. $(\mathbf{H})$ Comparison of distance between adjacent root hairs in a single vertical trichoblast cell file in WT (D) Atdjc17-1-1 (E) and overexpressor 35S::AtDjC17 (F). *indicates significant difference $(P \leq 0.05)$. $n=15)$ of the Atdjc17 mutant, which was consistent with a "scrambling" of expression among transcripts involved in epidermal patterning.

Transcriptional analyses of $A t D j C 17$ and 12 known regulators of root development (Figure 6) were investigated in the overexpressor of 35S::AtDjC17. As expected, 35S::AtDjC17 increased $A t D j C 17$ transcript levels but it was also noted a downregulation of GL2, which was consistent with the ectopic root hair phenotype previously described (Figure 1). In addition to
35S::AtDjC17 also the transcript levels of GL3, TTG2, and SCR were also up-regulated. Consistently with GL2 also CPC was found down-regulated along with $S H R$, whereas the remaining regulators were found to be unchanged.

\section{CORTICAL AND ENDODERMAL CELL LAYERS DISPLAY ABERRANT DIVISIONS IN Atdjc17 BUT NOT IN 35S::AtDjC17}

Phenotypes associated with the dysfunction in the stele expressed $S H R$, and ground tissue stem cell expressing MGP and JKD 


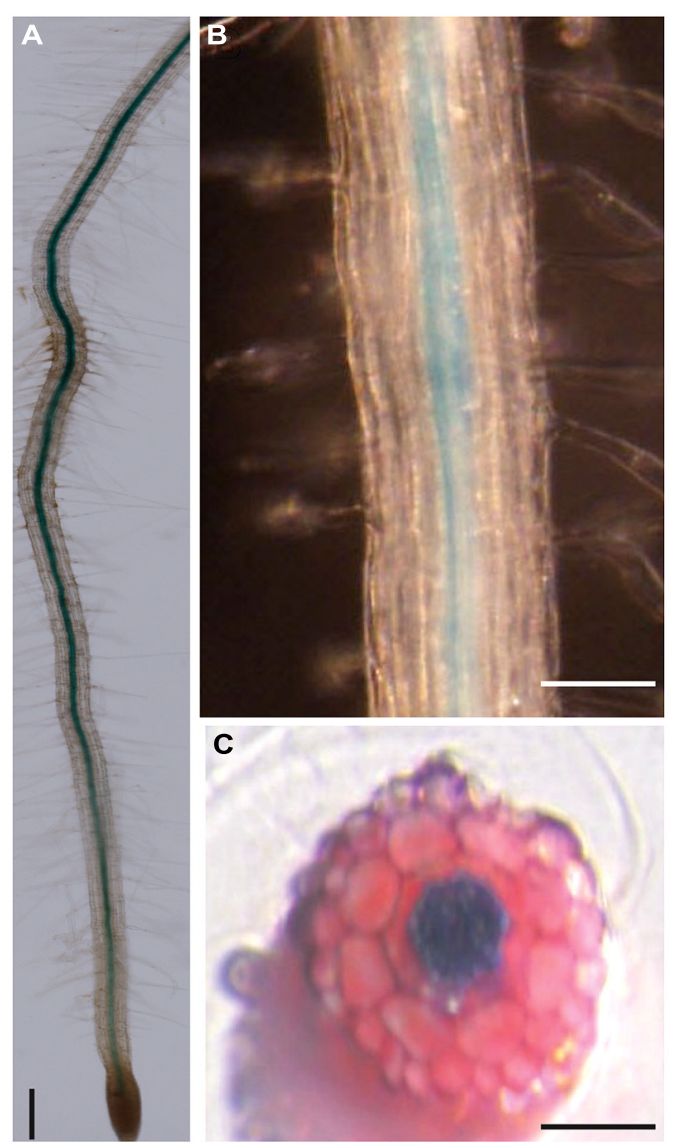

FIGURE 3 | GUS expression studies. (A,B) Stele-localized accumulation of proAtDjC17::GUS transcript in a 7-d old root. (A) Scale Bar $100 \mu \mathrm{m}$. (B) Scale Bar $50 \mu \mathrm{m}$. (C) Agarose embedded hand section of proAtDjC17::GUS expressing 7-d old roots displaying a clear stele accumulation of GUS, Scale Bar $50 \mu \mathrm{m}$

genes include irregular pattern formation in the cortical and endodermal cell layers (Helariutta et al., 2000; Welch et al., 2007; Koizumi et al., 2011; Ogasawara et al., 2011). Although shr has a far more severe effect on plant growth and development than what we have documented for Atdjc17 alleles (Figures 1 and 2; Supplementary material Figure S2) Atdjc17 subtle phenotypes are more similar to the one documented for $m g p$ and $j d w$. Using laser scanning confocal microscopy, we examined the longitudinal cell file development of Atdjc17 and WT using propidium iodide to fluorescently label the cell walls. Here, the cortical and endodermal cell layers in Atdjc17 root did not show obvious changes in cell division relative to WT (Figures 5D-F). By contrast, when we examined cross sections of WT, Atdjc17 and 35S::AtDjC17 seedling roots it was evident that the cortical (co) and endodermal (en) layer displayed increased frequency of cell divisions in the Atdjc17 mutants (co: 9 cells per section $\pm 1, n=15$; en: 9 cells per section \pm 1 , $n=15$ ) compared to the WT ( 8 cells per section $\pm 0, n=15$; Figures 5A,B). On the contrary, 35S::AtDjC17 did not show observable alteration in divisions in either cortical cells or endodermal cell layers (Figure 5C). Absence of visible alteration in the longitudinal propidium iodide stained section suggests an anticlinal division alteration only identifiable through cross sections. Changes in the number of cells in the cortical layer directly influences the frequency of trichoblast cells in the epidermal layer due to the position requirement for contact with two underlying cortical cells (Kwak and Schiefelbein, 2007), which was consistent with the observed aberrant H-cell occurrence visualized in Atdjc17 mutants (Figure 1). The modest changes in root patterning (increase cell division: Atdjc171-1/12: 9 cells/section \pm 1 vs. WT 8 cells/section $\pm 0, n=15$ ) and development (increase in cell frequency in endodermal layer Atdjc171-1/1-2: 9 cells/section \pm 1 vs. WT 8 cells/section \pm 0 , $n=15$ ) displayed similarity to $m g p$ and $j k d$ (Welch et al., 2007; Ogasawara et al., 2011).

\section{DISCUSSION}

Mutations in AtDjC17 identified in this study, caused aberrant cell fate determination and cell divisions in ground tissue layers in Arabidopsis roots. Existing literature supports DNAJ/J-domain family proteins functioning as co-chaperones working in association with HSP70 class proteins (Miernyk, 2001; Qiu et al., 2006; Summers et al., 2009; Jelenska et al., 2010; Bekh-Ochir et al., 2013) and based on the observed root phenotypes, we hypothesized that AtDjC17 would plausibly be required for cell fate determination by acting in a client:binary complex with a cognate HSP70 as a protein chaperone to fortify key stage(s) in the pathway. We envisioned that chaperone function could be important, particularly at the epidermis, where exposure to the adjacent soil environment may require such a chaperone due to environmental stress. However, an unexpected feature of the $A t D j C 17$ was its prominent expression in restricted to stele tissue of the Arabidopsis root (Figure 3). This is despite H-cell irregularity phenotypes being observed in the epidermis (Figure 1). Interestingly, dysfunction in genes encoding the zinc finger proteins $J K D$ and MGP resulted in a similar syndrome of defective epidermal cell fate determination and division defects despite transcription being localized outside of epidermal tissue. JKD expression is localized in ground tissue, quiescent center (QC) and to a lesser extent in mature cortical cells and is known to limit SHR and control cell divisions that give rise to endodermal and cortical layers (Welch et al., 2007; Hassan et al., 2010). $S H R$, a GRAS family transcription factor is expressed in stele tissue (Welch et al., 2007) and influences root development by influencing asymmetric divisions that give rise to ground tissue as well as endodermal cell identity. It does this in part by regulating SCR, another GRAS family transcription factor (Helariutta et al., 2000). Both SHR and SCR affect overall root development as mutations in these genes causes supernumerary and replacement of cortical/endodermal cell layer with a single ground cell layer having heterogeneous cell identity. Therefore, matching expression for AtDjC17 and SHR suggests possible functional influence in the same pathway. We propose a model whereby a chaperone complex involving AtDjC17 could be influencing this pathway in a non-cell autonomous fashion that involves $S H R, S C R, J K D$, and MGP.

Indeed, examining gene expression of SHR and SCR in Atdjc17 supports these data. Quantitative real time (Q-RT) PCR revealed 
A

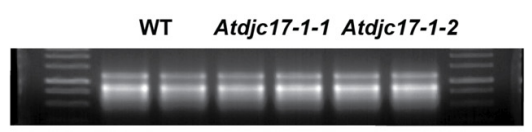

B

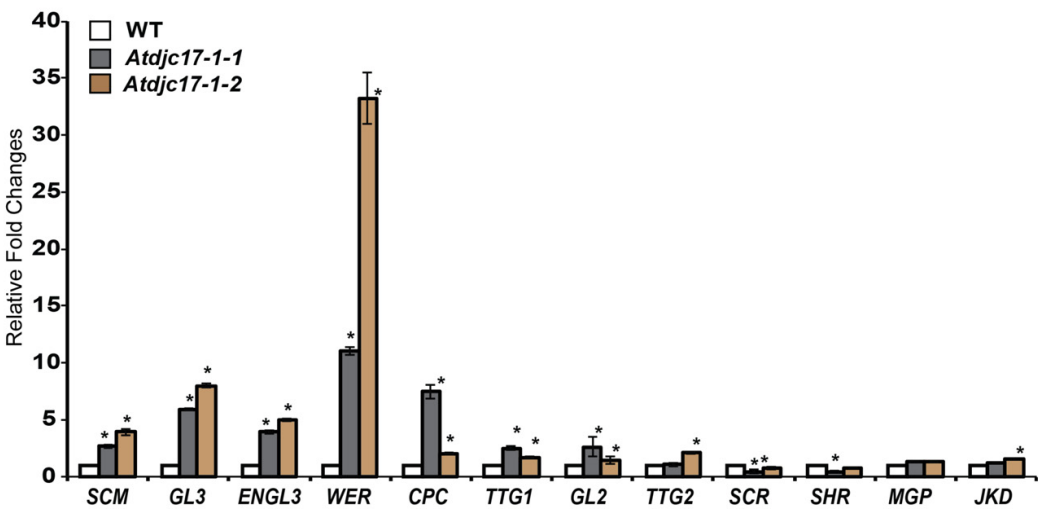

FIGURE 4 | Gene expression analysis for previously identified regulatory elements of root development. (A) RNA equal loading of WT and Atdjc17 mutant lines (Atdjc17-1-1, Atdjc17-1-2). (B) Relative fold changes determined on whole root sample for SCRAMBLE (SCM); GLABRA3 (GL3); Enhancer of GLABRA3 (ENGL3);WEREWOLF
(WER); CAPLICE (CPC); TRANSPARENT TESTA 1 (TTG1);GLABRA2 (GL2): TRANSPARENT TESTA 2 (TTG2); SCARECROW (SCR):

SHORTROOT (SHR); MAGPIE (MGP) and JAKDOW (JKD). Error bars indicate standard deviation. *indicates significant difference $(P \leq 0.05)$
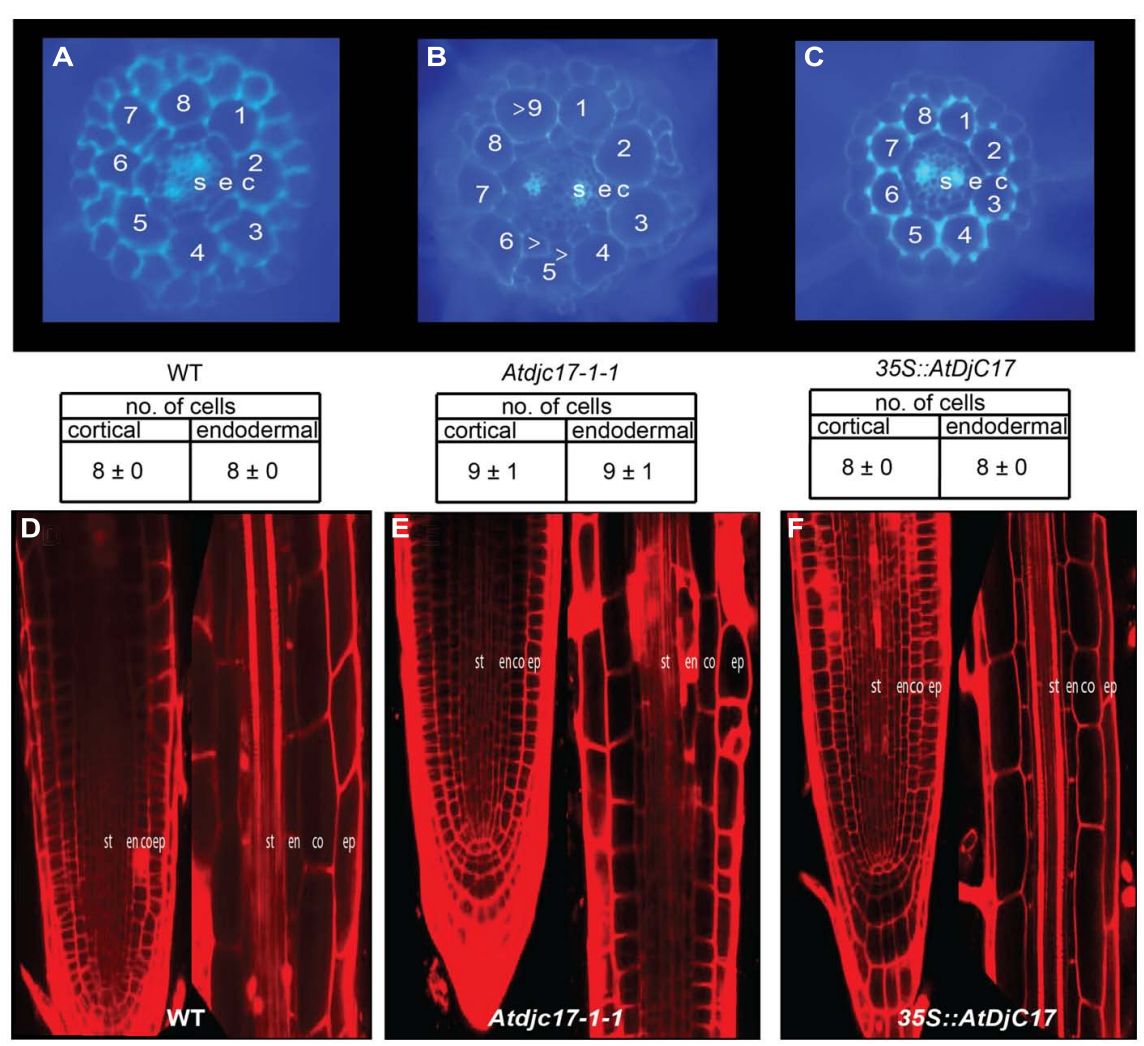

FIGURE 5 | Examination of cell division in WT, Atdjc171-1 and 35S::AtDjC17 roots: agarose embedded hand sections stained with calcofluor-white stained and confocal microscopic images of propidium iodide stained WT, Atdjc17-1-1 and 35S::AtDjC17 mutant roots. (A) WT section. (B) Atdjc17-1-1 section showing additional cell division in the cortical and endodermal layers. Note that the divisional pattern seems anticlinal in nature. Arrow heads indicate ectopic divisions. (C) 35S::AtDjC17 section with no evidence of altered cortical and endodermal cell numbers. (D) 7-day old post-germination WT root tip and zone above the meristematic tip in WT root. (E) Atdjc17-1-1 root tip with associated zone above the meristematic tip. (F) 35S::AtDjC17 root tip and elongation zone. st, stele; en, endodermis; co, cortex; ep, epidermis. 


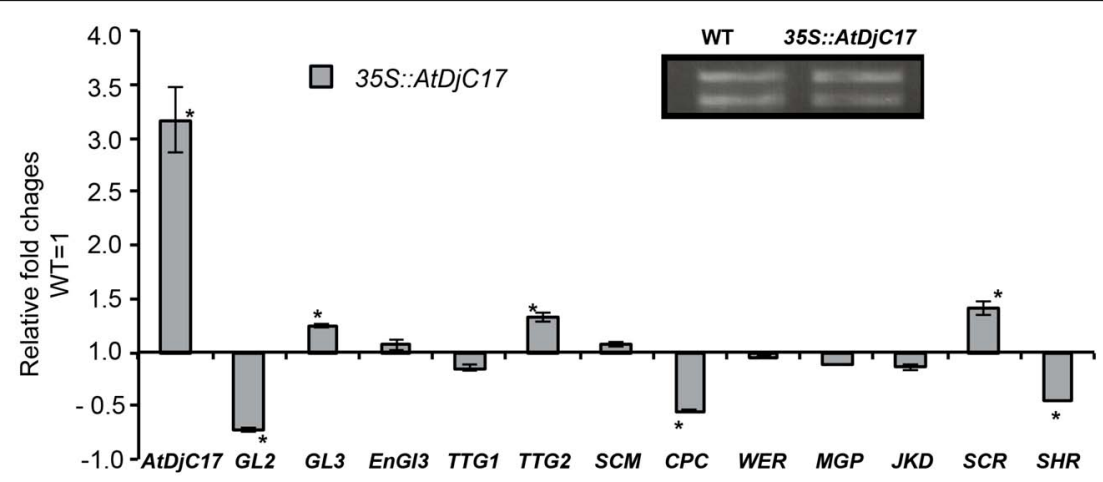

FIGURE 6 | Root expression analysis of the overexpressor of $\boldsymbol{A t D j C 1 7}$. Relative fold changes determined on whole root sample for $35 \mathrm{~S}:$ :AtDjC17 and 12 main regulators of root development. Error bars indicate standard deviation. ${ }^{*}$ indicates significant difference $(P \leq 0.05)$.

down-regulation of $S C R$. We anticipated that down-regulation of $S C R$ in Atdjc17 would be mirrored by SHR, which was confirmed by Q-RT PCR (Figure 4). By contrast, in the whole root samples from Atdjc17, trichoblast, and atrichoblast specific expressed transcripts [atrichoblast (WER, GL2, CPC); trichoblast (EGL3, $G L 3$, )] both displayed increased abundance relative to WT. Considering the mixed identity among epidermal cell files observed as H-cell-fate irregularities, the observed increased transcript abundance among these cell type specific transcripts was not unexpected, but does not conclusively suggest any single element as responsive. Alternatively, Q-RT PCR on whole root samples might not be a sensitive method to uncover differences in cell type specific transcript levels. Cell type specific Q-RT PCR on Atdjc17 may provide better understanding of the differences in gene expression for epidermal patterning specific genes.

Intriguingly, expression levels of $J K D$ and $M G P$, were not differentially expressed in Atdjc17 further suggests that AtDjC17 functions independently of these factors. These data did not support our hypothesis of transcriptional linkage to the zinc finger proteins $J K D$ and $M G P$ but did identify transcriptional association to $S C R / S H R$. Given its critical requirement for root development, it is indeed plausible that molecular chaperone function for $S H R$ would be important to safeguard the root developmental program. Taken together, JKD null mutations cause ectopic periclinal divisions in the cortical and endodermal layer but also an ectopic root hair development in a non-cell autonomous fashion (Welch etal., 2007; Hassan et al., 2010). Hence, while the localization of AtDjC17 expression to stele cells partially overlapped with SHR the phenotype of Atdjc17 more closely resembled $j k d$, although with a far less severe impact on cell division and whole plant morphogenic phenotypes. The position dependence needed to acquire $\mathrm{H}$-cell versus $\mathrm{N}$-cell fate in epidermal cells (Dolan, 2006) is such that ectopic divisions within the cortical cells observed in transverse cross sections of the Atdjc17 root could explain the corresponding irregular pattern of H-cell emergence (Figure 1). Further studies are needed to assign biochemical association between possible targets of the AtDjC17 co-chaperone, in addition to isolation of the anticipated cognate HSP70. Although many genes have been identified in root development (Guimil and Dunand, 2006) no prior evidence supports a requirement for a DNAJ-HSP40 for epidermal cell fate determination, and results from Atdjc17 raise the intriguing possibility of a HSP complex playing a chaperone role in root development.

\section{AUTHOR CONTRIBUTIONS}

Carloalberto Petti carried out the phenotyping and genotyping of the T-DNA, the RT analyses and the transgenesis and drafted the manuscript. Meera Nair helped with the phenotyping and genotyping of the T-DNA lines and drafted the manuscript. Seth DeBolt conceived the study and drafted the manuscript. All authors read and approved the final manuscript.

\section{ACKNOWLEDGMENTS}

We thank Jozsef Stork and Jordan Clay (University of Kentucky) for technical assistance. This work was supported by the National Science Foundation (NSF) MCB 1122016 and NSF EPSCoR.

\section{SUPPLEMENTARY MATERIAL}

The manuscript contains a supplementary table (Table S1) listing the primers employed for genotyping, cloning, and for RT-PCR studies. Additionally it also contains three supplementary figures displaying the position of the insertion (Figure S1); detailed characterization of root hair phenotype of Atdjc17-1-2 (Figure S2); complemented T-DNA lines by native promoter driven construct (Figure S3). The Supplementary Material for this article can be found online at: http://www.frontiersin.org/journal/10.3389/fpls.2014.00532/ abstract

\section{REFERENCES}

Al-Whaibi, M. (2011). Plant heat-shock proteins: a mini review. J. King Saud Univ. Sci. 23, 139-150. doi: 10.1016/j.jksus.2010.06.022

Assaad, F., Huet, Y., Mayer, U., and Jürgens, G. (2001). The cytokinesis gene KEULE encodes a Secl protein that binds the syntaxin KNOLLE. J. Cell Biol. 152, 531-543. doi: $10.1083 /$ jcb.152.3.531

Bakau, B., and Horwich, A. L. (1998). The Hsp70 and Hsp60 chaperone machines. Cell 92, 351-366. doi: 10.1016/S0092-8674(00)80928-9

Bekh-Ochir, D., Shimada, S., Yamagami, A., Kanda, S., Ogawa, K., Nakazawa, M., et al. (2013). A novel mitochondrial DnaJ/Hsp40 family protein BIL2 promotes 
plant growth and resistance against environmental stress in brassinosteroid signaling. Planta 237, 1509-1525. doi: 10.1007/s00425-013-1859-3

Bernhardt, C., Zhao, M., Gonzalez, A., Lloyd, A., and Schiefelbein, J. (2005). The bHLH genes GL3 and EGL3 participate in an intercellular regulatory circuit that controls cell patterning in the Arabidopsis root epidermis. Development 132, 291298. doi: 10.1242/dev.01565

Brady, S. M., Orlando, D. A., Lee, J. Y., Wang, J. Y., Koch, J., Dinneny, J. R., et al. (2007). A high-resolution root spatiotemporal map reveals dominant expression patterns. Science 318, 801-806. doi: 10.1126/science.1146265

Bruex, A., Kainkaryam, R. M., Wieckowski, Y., Kang, Y. H., Bernhardt, C., Xia Y., et al. (2012). A gene regulatory network for root epidermis cell differentiation in Arabidopsis. PLoS Genet. 8:e1002446. doi: 10.1371/journal.pgen. 1002446

Cao, X., Linstead, P., Berger, F., Kieber, J., and Dolan, L. (1999). Differential ethylene sensitivity of epidermal cells is involved in the establishment of cell pattern in the Arabidopsis root. Physiol. Plant. 106, 311-317. doi 10.1034/j.1399-3054.1999.106308.x

Chen, S., Songkumarn, P., Liu, J., and Wang, G. L. (2009). A versatile zero background T-vector system for gene cloning and functional genomics. Plant Physiol. 150, 1111-1121. doi: 10.1104/pp.109.137125

Clough, S., and Bent, A. (1998). Floral dip: a simplified method for Agrobacterium-mediated transformation of Arabidopsis thaliana. Plant J. 16, 735-743. doi: 10.1046/j.1365-313x.1998.00343.x

Di Laurenzio, L., Wysocka-Diller, J., Malamy, J. E., Pysh, L., Helariutta, Y., Freshour, G., et al. (1996). The SCARECROW gene regulates an asymmetric cell division that is essential for generating the radial organization of the Arabidopsis root. Cell 86, 423-433. doi: 10.1016/S0092-8674(00)80115-4

Dolan, L. (2006). Positional information and mobile transcriptional regulators determine cell pattern in the Arabidopsis root epidermis. J. Exp. Bot. 57, 51-54. doi: $10.1093 / \mathrm{jxb} / \mathrm{erj037}$

Dolan, L., Duckett, C. M., Grierson, C., Linstead, E., Schneider, K., Lawson, E., et al. (1994). Clonal relationships and cell patterning in the root epidermis of Arabidopsis. Development 120, 2465-2474.

Galway, M. E., Masucci, J. D., Lloyd, A. M., Walbot, V., Davis, R. W., and Schiefelbein, J. W. (1994). The TTG gene is required to specify epidermal cell fate and cell patterning in the Arabidopsis root. Dev. Biol. 166, 740-754. doi 10.1006/dbio.1994.1352

Guimil, S., and Dunand, C. (2006). Patterning of Arabidopsis epidermal cells: epigenetic factors regulate the complex epidermal cell fate pathway. Trends Plant Sci. 11, 601-609. doi: 10.1016/j.tplants.2006.10.001

Guivarc'h, A., Caissard, J. C., Azmi, A., Elmayan, T., Chequi, D., and Tepfer, M. (1996). In situ detection of expression of GUS reporter gene in transgenic plants: ten years of blue genes. Transgenic Res. 5, 281-298. doi: 10.1007/BF019 68938

Hassan, H., Scheres, B., and Blilou, I. (2010). JACKDAW controls epidermal patterning in the Arabidopsis root meristem through a non-cell-autonomous mechanism. Development 137, 1523-1529. doi: 10.1242/dev.048777

Helariutta, Y., Fukaki, H., Wysocka-Diller, J., Nakajima, K., Jung, J., Sena, G., et al. (2000). The SHORT-ROOT gene controls radial patterning of the Arabidopsis root through radial signaling. Cell 101, 555-567. doi: 10.1016/S0092-8674(00) 80865-X

Hung, C. Y., Lin, Y., Zhang, M., Pollock, S., David Marks, M., and Schiefelbein, J. (1998). A common position-dependent mechanism controls cell-type patterning and GLABRA2 regulation in the root and hypocotyl epidermis of Arabidopsis. Plant Physiol. 117, 73-84. doi: 10.1104/pp.117.1.73

Ishida, T., Hattori, S., Sano, R., Inoue, K., Shirano, Y., Hayashi, Y., et al. (2007). Arabidopsis TRANSPARENT TESTA GLABRA2 is directly regulated by R2R3 MYB transcription factors and is involved in regulation of GLABRA2 transcription in epidermal differentiation. Plant Cell 19, 2531-2543. doi: 10.1105/tpc.107.052274

Ishida, T., Kurata, T., Okada, K., and Wada, T. (2008). A genetic regulatory network in the development of trichomes and root hairs. Annu. Rev. Plant Biol. 59, 365-386. doi: 10.1146/annurev.arplant.59.032607.092949

Jefferson, R. A., Kavanagh, T. A., and Bevan, M. W. (1987). GUS fusions: $\beta$ glucuronidase as a sensitive and versatile gene fusion marker in higher plants. EMBO J. 6, 3901-3907.

Jelenska, J., van Hal, J. A., and Greenberg, J. T. (2010). Pseudomonas syringae hijacks plant stress chaperone machinery for virulence. Proc. Natl. Acad. Sci. U.S.A. 107, 13177-13182. doi: 10.1073/pnas.0910943107
Jones, M., Shen, J., Fu, Y., Li, H., Yang, Z., and Grierson, C. (2002). The Arabidopsis Rop2 GTPase is a positive regulator of both root hair initiation and tip growth. Plant Cell 14, 763-776. doi: 10.1105/tpc.010359

Jones, M. A., Raymond, M. J., and Smirnoff, N. (2006). Analysis of the roothair morphogenesis transcriptome reveals the molecular identity of six genes with roles in root-hair development in Arabidopsis. Plant J. 45, 83-100. doi: 10.1111/j.1365-313X.2005.02609.x

Knox, K., Grierson, C., and Leyser, O. (2003). AXR3 and SHY2 interact to regulate root hair development. Development 130, 5769-5777. doi: 10.1242/dev.00659

Koizumi, K., Wu, S., MacRae-Crerar, A., and Gallagher, K. L. (2011). An essentail protein that interacts with endosomes and promotes movement of the SHORT-ROOT transcription factor. Curr. Biol. 21, 1559-1564. doi: 10.1016/j.cub.2011.08.013

Koshino-Kimura, Y., Wada, T., Tachibana, T., Tsugeki, R., Ishiguro, S., and Okada, K. (2005). Regulation of CAPRICE transcription by MYB proteins for root epidermis differentiation in Arabidopsis. Plant Cell Physiol. 46, 817-826. doi: $10.1093 / \mathrm{pcp} / \mathrm{pci096}$

Kregel, K. C. (2002). Heat shock proteins: modifying factors in physiological stress responses and acquired thermotolerance. J. Appl. Physiol. 122, 189-197.

Kwak, S. H., and Schiefelbein, J. (2007). The role of the SCRAMBLED receptor-like kinase in patterning the Arabidopsis root epidermis. Dev. Biol. 302, 118-131. doi: 10.1016/j.ydbio.2006.09.009

Kwak, S. H., and Schiefelbein, J. (2008). A feedback mechanism controlling SCRAMBLED receptor accumulation and cell-type pattern in Arabidopsis. Curr. Biol. 18, 1949-1954. doi: 10.1016/j.cub.2008.10.064

Kwak, S. H., Shen, R., and Schiefelbein, J. (2005). Positional signaling mediated by a receptor-like kinase in Arabidopsis. Science 307, 1111-1113. doi: $10.1126 /$ science. 1105373

Lee, M. M., and Schiefelbein, J. (1999). WEREWOLF, a MYB-related protein in Arabidopsis, is a position-dependent regulator of epidermal cell patterning. Cell 99, 473-483. doi: 10.1016/S0092-8674(00)81536-6

Lee, M. M., and Schiefelbein, J. (2002). Cell pattern in the Arabidopsis root epidermis determined by lateral inhibition with feedback. Plant Cell 14, 611-618. doi: $10.1105 /$ tpc. 010434

Masucci, J., Rerie, W., Foreman, D., Zhang, M., Galway, M., Marks, M., et al. (1996). The homeobox gene GLABRA2 is required for position-dependent cell differentiation in the root epidermis of Arabidopsis thaliana. Development 122, 1253-1260.

Miernyk, J. A. (2001). The J-domain proteins of Arabidopsis thaliana: an unexpectedly large and diverse family of chaperones. Cell Stress Chaperones 6, 209-218. doi: 10.1379/1466-1268(2001)006<0209:TJDPOA >2.0.CO;2

Minami, Y., Höhfeld, J., Ohtsuka, K., and Hartl, F. U. (1996). Regulation of the heat-shock protein 70 reaction cycle by the mammalian DnaJ homolog hsp40. J. Biol. Chem. 271, 19617-19624. doi: 10.1074/jbc.271.32.19617

Mylona, P., Linstead, P., Martienssen, R., and Dolan, L. (2002). SCHIZORIZA controls an asymmetric cell division and restricts epidermal identity in the Arabidopsis root. Development 129, 4327-4334.

Nagpal, P., Walker, L., Young, J., Sonawala, A., Timpte, C., Estelle, M., et al. (2000). AXR2 encodes a member of the Aux/IAA protein family. Plant Physiol. 123, 563-574. doi: 10.1104/pp.123.2.563

Nakamoto, H., and Vígh, L. (2007). The small heat shock proteins and their clients. Cell. Mol. Life Sci. 64, 294-306. doi: 10.1007/s00018-006-6321-2

Nawy, T., Lee, J. Y., Colinas, J., Wang, J. Y., Thongrod, S. C., Malamy, J. E., et al. (2005). Transcriptional profile of the Arabidopsis root quiescent center. Plant Cell 17, 1908-1925. doi: 10.1105/tpc.105.031724

Ogasawara, H., Kaimi, R., Colasanti, J., and Kozaki, A. (2011). Activity of transcription factor JACKDAW is essential for SHR/SCR-dependent activation of SCARECROW and MAGPIE and is modulated by reciprocal interactions with MAGPIE, SCARECROW, and SHORT ROOT. Plant Mol. Biol. 77, 489-499. doi: 10.1007/s11103-011-9826-5

Panaretou, B., and Zhai, C. (2008). The heat shock proteins: their roles as multicomponent machines for protein folding. Fungal Biol. Rev. 22, 110-119. doi: 10.1016/j.fbr.2009.04.002

Pechan, P. (1991). Heat shock proteins and cell proliferation. FEBS Lett. 280, 1-4. doi: 10.1016/0014-5793(91)80190-E

Qiu, X. B., Shao, Y. M., Miao, S., and Wang, L. (2006). The diversity of the dnaJ/Hsp40 family, the crucial partners for Hsp70 chaperones. Cell. Mol. Life Sci. 63, 2560-2570. doi: 10.1007/s00018-006-6192-6 
Rajan, V. B., and D'Silva, P. (2009). Arabidopsis thaliana J-class heat shock proteins: cellular stress sensors. Funct. Integr. Genomics 9, 433-446. doi: 10.1007/s10142009-0132-0

Ritossa, F. (1962). A new puffing pattern induced by heat shock and DNP in Drosophila. Experientia 18, 571-573. doi: 10.1007/BF02172188

Rogers, S. O., and Bendich, A. J. (1985). Extraction of DNA from milligram amounts of fresh, herbariurm and mummified plant tissues. Plant Mol. Biol. 5, 69-76. doi: 10.1007/BF00020088

Sabatini, S., Heidstra, R., Wildwater, M., and Scheres, B. (2003). SCARECROW is involved in positioning the stem cell niche in the Arabidopsis root meristem. Genes Dev. 17, 354-358. doi: 10.1101/gad. 252503

Schellmann, S., Schnittger, A., Kirik, V., Wada, T., Okada, K., Beermann, A., et al. (2002). TRIPTYCHON and CAPRICE mediate lateral inhibition during trichome and root hair patterning in Arabidopsis. EMBO J. 21, 5036-5046. doi: 10.1093/emboj/cdf524

Siddique, M., Gernhard, S., von Koskull-Döring, P., Vierling, E., and Scharf, K. (2008). The plant sHSP superfamily: five new members in Arabidopsis thaliana with unexpected properties. Cell Stress Chaperones 13, 183-197. doi: 10.1007/s12192-008-0032-6

Summers, D. W., Douglas, P. M., Ramos, C. H. I., and Cyr, D. M. (2009). Polypeptide transfer from Hsp40 to Hsp70 molecular chaperones. Trends Biochem. Sci. 34, 230-233. doi: 10.1016/j.tibs.2008.12.009

ten Hove, C. A., Willemsen, V., de Vries, W. J., van Dijken, A., Scheres, B., and Heidstra, R. (2010). SCHIZORIZA encodes a nuclear factor regulating asymmetry of stem cell divisions in the Arabidopsis root. Curr. Biol. 20, 452-457. doi: 10.1016/j.cub.2010.01.018

Tissiers, A., Mitchell, H. K., and Tracy, U. M. (1974). Protein synthesis in salivary glands of D. melanogaster. Relation to chromosome puffs. J. Mol. Biol. 84, 249-258.

Wada, T., Kurata, T., Tominaga, R., Koshino-Kimura, Y., Tachibana, T., Goto, K., et al. (2002). Role of a positive regulator of root hair development, CAPRICE, in Arabidopsis root epidermal cell differentiation. Development 129, 5409-5419. doi: 10.1242/dev.00111

Wada, T., Tachibana, T., Shimura, Y., and Okada, K. (1997). Epidermal cell differentiation in Arabidopsis determined by a myb homolog, CPC. Science 277, 1113-1116. doi: 10.1126/science.277.5329.1113

Walsh, P., Bursać, D., Law, Y., Cyr, D., and Lithgow, T. (2004). The J-protein family: modulating protein assembly, disassembly and translocation. EMBO Rep. 5, 567571. doi: 10.1038/sj.embor.7400172

Welch, D., Hassan, H., Blilou, I., Immink, R., Heidstra, R., and Scheres, B. (2007). Arabidopsis -JACKDAW, and MAGPIE zinc finger proteins delimit asymmetric cell division, and stabilize tissue boundaries by restricting SHORT-ROOT action. Genes Dev. 21, 2196-2204. doi: 10.1101/gad.440307

Zhang, F., Gonzalez, A., Zhao, M., Payne, C. T., and Lloyd, A. (2003). A network of redundant bHLH proteins functions in all TTG1-dependent pathways of Arabidopsis. Development 130, 4859-4869. doi: 10.1242/dev.00681

Conflict of Interest Statement: The authors declare that the research was conducted in the absence of any commercial or financial relationships that could be construed as a potential conflict of interest.

Received: 25 July 2014; paper pending published: 28 August 2014; accepted: 18 September 2014; published online: 08 October 2014.

Citation: Petti C, Nair M and DeBolt S (2014) The involvement of J-protein AtDjC17 in root development in Arabidopsis. Front. Plant Sci. 5:532. doi: 10.3389/fpls.2014.00532 This article was submitted to Plant Physiology, a section of the journal Frontiers in Plant Science.

Copyright (C) 2014 Petti, Nair and DeBolt. This is an open-access article distributed under the terms of the Creative Commons Attribution License (CC BY). The use, distribution or reproduction in other forums is permitted, provided the original author(s) or licensor are credited and that the original publication in this journal is cited, in accordance with accepted academic practice. No use, distribution or reproduction is permitted which does not comply with these terms. 